\section{After Cesarean vesicouterine fistula (Youssef Syndrome) with successful conservative management}

\author{
Julián Oñate Celdrán, ${ }^{1}$ \\ Francisco M. Gonzalez Valverde ${ }^{2}$ \\ ${ }^{1}$ Department of Urology; and \\ ${ }^{2}$ Department of Surgery, Pediatrics and \\ Obstetrics and Gynecology, Reina Sofia \\ General University Hospital, Murcia. \\ Spain
}

\begin{abstract}
Vesicouterine fistula without vaginal leakage of urine, cyclic hematuria and amenorrhea is a rare condition, identified as Youssef's syndrome. The most common cause of this syndrome is trauma during a cesarean section. Different therapeutical approaches that include conservative treatment, fulguration, hormonal therapy and open, laparoscopic or robotic surgeries have been mentioned in the literature. Herein a case in a 37-year-old patient is reported. Conservative treatment with urinary drainage was successful because the fistula was recognized in the immediate postoperative period.
\end{abstract}

\section{Introduction}

Youssef's syndrome, characterized by vesicouterine fistula (VUF) without vaginal leakage of urine, cyclic hematuria (menouria) and amenorrhea ${ }^{1}$ is a rare condition caused in most cases by obstetric procedures, especially emergency Cesarean delivery. ${ }^{2}$ We report a case of VUF treated successfully with urinary drainage.

\section{Case Report}

A 37-year-old woman (gravida 2, para 2 ), with a history of one cesarean birth three years before, presented with hematuria after vaginal delivery 3 weeks early. Urine cultures were sterile and laboratory data established anemia (9.3 g/dL), mild leukocytosis; a white cell count of $12.1 \times 10^{9} / \mathrm{L}$, neutrophils of $8.4 \times 10^{9} / \mathrm{L}$ and a $\mathrm{C}$ reactive protein of $21 \mathrm{mg} /$ catheterization of the bladder yielded clear urine. Cystoscopy demonstrated an edematous area around a pinhole fistula at the bladder dome (Figure 1A). Methylene blue, which was injected into the bladder, was readily discharged from the vagina. Dehiscence of the previous Cesarean scar with formation of a VUF was the initial diagnosis. Computed tomography (CT) revealed a $2 \mathrm{~mm}$ fistulous tract communicating the bladder dome with the corpus uteri (Figure 1B), which was consistent with VUF.

Conservative management with transurethral catheter was instituted. Urine drainage prolonged for two months, which resulted in complete resolution of both clinical and radiographic findings. Cystoscopy control revealed the closure of the fistula and at the second year of follow-up the patient remains asymptomatic.

\section{Discussion and Conclusions}

Vesicouterine fistula without vaginal leakage of urine due to the sphincteric mechanism of the cervix was first described by Youssef in $1957 .{ }^{1}$ This rare condition represents only $1-4 \%$ of all urogenital fistulas, although currently their prevalence is increasing for the frequent use of the cesarean section (CS). ${ }^{2}$ After CS the bladder gets so adhered to the uterus that it is subjected to severe tension during a following vaginal delivery, which may result in VUF formation. ${ }^{3}$ Clinical presentations include urinary incontinence, cyclic hematuria due to menstruation through the bladder and amenorrhea. Recurrent urinary infections, secondary infertility and first trimester abortions are less common reasons for consultation. ${ }^{2,3}$ VUF usually generate psychological distress and have a negative effect on quality of life.

The bladder may be damaged by direct injury, inadequate mobilization or aberrant suture $^{3}$. This can be prevented by emptying the bladder and by carefully dissecting the lower uterine segment. Porcaro ${ }^{4}$ propose intraoperative sonography by the transvaginal (or transrectal) route when suspecting bladder injury while dissecting the uterine lower segment and for monitoring patients who already had a VUF repair. Moreover, ultrasound Doppler examination may help in better investigating and understanding the pathophysiology of VUF.

Usually, VUF are discovered postoperatively. The accurate diagnosis is ruled out by showing the fistulous track by cystoscopy, retrograde cystography and hysterosalpingography. 5,6 Methylene blue test, $\mathrm{CT}$ and magnetic resonance imaging (MRI), which are valuable for diagnosing vesicovaginal fistula, must also warrant consideration. Cystography and hysterography shows the fistulous track as an abnormal communication between the uterus and the bladder. ${ }^{6}$ Cystoscopy shows irregular hole
Correspondence: Francisco Miguel González Valverde, Hospital General Universitario Reina Sofía de Murcia, Intendente Jorge Palacios 1. CP: 30.003. Murcia, Spain.

Tel.: 968359000 - Fax: 968359655.

E-mail:migova67@gmail.com

Key words: Cesarean section; uterovesical fistula; Youssef's syndrome; bladder fistula; urinary incontinence.

Contributions: both authors have made a significant contribution to the findings and methods in the paper: JOC, conception and design, material support, acquisition of data and final approval of the version to be published; FMGV, Supervision, analysis and interpretation of data, technical support, drafting of the manuscript and final approval of the version to be published.

Received for publication: 24 April 2017.

Revision received: 14 September 2017.

Accepted for publication: 22 September 2017.

This work is licensed under a Creative Commons Attribution NonCommercial 4.0 License (CC BY-NC 4.0).

(C) Copyright J. Oñate Celdrán and F.M. Gonzalez Valverde, 2017

Licensee PAGEPress, Italy

Urogynaecologia 2017; 30:199

doi:10.4081/uij.2017.199

or depression, which may be surrounded by edema and inflammation of the urothelial mucosa. CT and MRI may show the patency of the fistula. ${ }^{7}$

The therapeutical approaches include conservative treatment as well as open surgery. ${ }^{3,4,8,9}$ Traditional treatment of VUF is surgical closure via laparotomy, but a few cases of spontaneous closure of fistula caused by Cesarean section have been reported although the size of the VUFs was not documented..$^{9,10}$ Conservative management with bladder catheterization, antibiotics, anticholinergics and induction of amenorrhea can be successful when, as in the presented case, the fistula is diagnosed within the first few days of surgery. ${ }^{4,10}$ If the fistula is not bigger than $0.5-1 \mathrm{~cm}$, it has a high spontaneous resolve or decrease rate with a 4-8 weeks trial of continued catheter drainage. If finished this term, the VUF has diminished in size; a trial of Foley drainage for an additional 2-3 weeks may be helpful. If no improvement is observed after a threemonth follow-up, a VUF is not likely to resolve conservatively. Under these circumstances, prolonged catheterization only increases the risks of infection and offers no increased benefit to fistula period. 

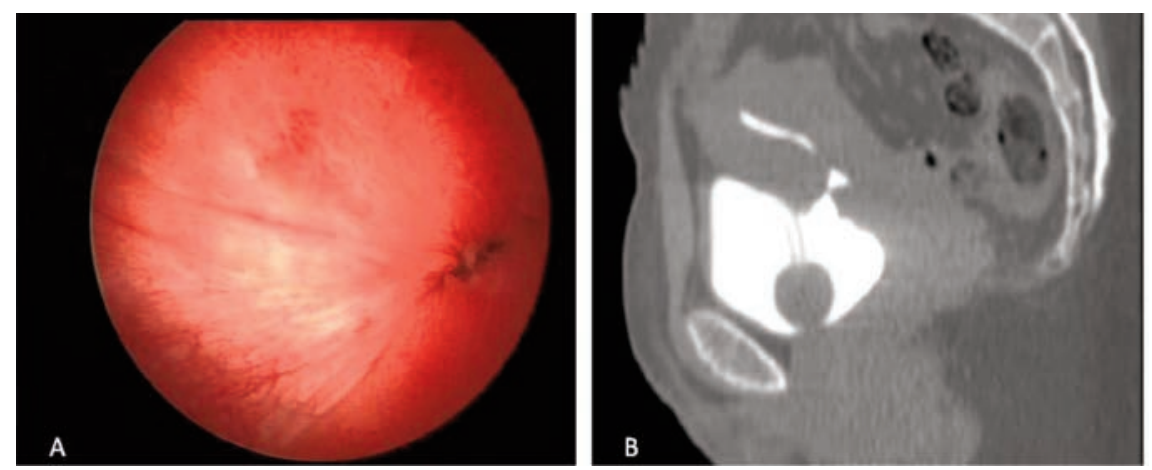

Figure 1. A) Cystoscopy showed a pinhole fistula at the posterior aspect of the bladder dome of the urinary bladder. B) Computed tomography of a sagittal view showed a communication between the urinary bladder and the uterus.

Spontaneous closure of the fistula in up to $5 \%$ of cases may be expected with the involution of the uterus. ${ }^{10}$ The ideal length of treatment is unknown as such as the adequate starting time, but this technique may be less successful in women with a mature tract (6 weeks or longer).

A proportion of this rare complication has been regarded as a certain type of endometriosis, which can provide an explanation for the efficacy of hormonal manipulation with contraceptive steroids. A combination of levonorgestrel $0.25 \mathrm{mg}$ and ethinyl estradiol $0.05 \mathrm{mg}$ daily for at least 6 months to induce amenorrhea may benefit patients with a small, well epithelialized orifice by the ectopic endometrial atrophy but a large established fistulous tract variably necessitates surgical repair. ${ }^{11}$ If hormone therapy fails, we would have to consider surgical treatment including less invasive procedures such as endoscopic fulguration of the fistula, which obtains spontaneous closure by destroying the ectopic endometrium, ${ }^{12}$ laparoscopic surgery ${ }^{13}$ or more recently, a robot-assisted laparoscopic repair. ${ }^{14}$

If the injury is discovered while performing surgery, it should be immediately repaired. ${ }^{5}$ Surgical repair may be performed 3 or 4 months after CS, because uterine involution has taken place and reaction to initial surgery should be minimal. Surgical repair of VUF could be performed by different approaches such vaginal or transvesicalretroperitoneal but we prefer the transperitoneal route, which allows a satisfactory mobilization of both bladder and uterus. ${ }^{5,9}$ Open bladder surgery together with hysterectomy is also a choice of treatment, particularly for multiparous patients.

Surgery is the mainstay and definitive treatment of VUF after CS but we think that conservative treatment should be tried first in small VUF discovered just after delivery. Bladder injury should be part of discussion with patients requesting elective CS.

\section{References}

1. Youssef AF. 'Menouria' following lower segment cesarean section: a syndrome. Am J Obstet Gynecol 1957;73:759.

2. Karaltı MO, Tınar Ş, Öztürk NT, Öztürk DC. A case with vesicouterine fistula: mini review. Arch Gynecol Obstet 2012;285:667-70.

3. Yip SK, Leung TY, Vesicouterine fistula: an updated review. Int Urogynecol J 1998;9:252-6.

4. Porcaro AB, Zicari M, Zecchini Antoniolli S, et al. Vesicouterine fistu- las following Caesarean section: report on a case, review and update of the literature. Int Urol Nephrol 2002;34:33544.

5. Lenkovsky Z, Pode D, Shapiro A, Caine M. Vesicouterine fistula: a rare complication of cesarean section. J Urol 1988;139:123-5.

6. Goel A, Goel S, Singh BP, Sankhwar SN. Cystographic images of Youssef syndrome: flower on top of the bladder. Urology 2012;79:e69-70.

7. Abou-El-Ghar ME, El-Assmy AM, Refaie HF, El-Diasty TA. Radiological diagnosis of vesicouterine fistula: role of magnetic resonance imaging. J Magn Reson Imaging 2012;36:438-42.

8. Perveen K, Al-Shaikh G, Al-Moazin M, et al. Urogenital fistula in a Saudi Arabian referral center. Arch Gynecol Obstet 2013;287:261-5.

9. Jozwik M, Jozvik M. Spontaneous closure of vesicouterine fistula. Account for effective hormonal treatment. Urol Int 1999;62:183-7.

10. Eogan M, McKenna P. Conservative management of a traumatic uterovesical fistula ('Youssef's syndrome'). Eur J Obstet Gynecol Reprod Biol 2003;10;110:114-6.

11. Yokoyama M, Arisawa C, Ando M. Successful management of vesicouterine fistula by luteinizing hormonereleasing hormone analog. Int $\mathrm{J}$ Urol 2006;13: 457-9.

12. Molina LR, Lynne CM, Politano VA. Treatment of vesicouterine fistula by fulguration. J Urol 1989;141:1422-3.

13. Unger CA, Cohen SL, Einarsson JI, Morse AN. Laparoscopic repair of vesicouterine fistula. Female Pelvic Med Reconstr Surg 2012;18:190-2.

14. Perveen K, Gupta R, Al-Badr A, Hemal AK. Robot-assisted laparoscopic repair of rare post-cesarean section vesicocervical and vesicouterine fistula: a case series of a novel technique. Urology 2012;80:477-82. 